Check for updates

Cite this: RSC Adv., 2017, 7, 43866

Received 6th June 2017

Accepted 16th August 2017

DOI: $10.1039 / c 7 r a 06312 a$

rsc.li/rsc-advances

\section{He-Wei granules inhibit chemotherapy-induced vomiting (CINV) in rats by reducing oxidative stress and regulating $5-H T$, substance $P$, ghrelin and obestatin $\uparrow$}

\author{
Zehai Song, ${ }^{\text {ab }}$ Hang Chang, ${ }^{a} \mathrm{Na} \mathrm{Han},{ }^{\mathrm{a}}$ Zhihui Liu, ${ }^{\mathrm{a}}$ Zhonglin Wang, ${ }^{\mathrm{a}} \mathrm{Hao}$ Gao \\ and Jun Yin (D) *ab
}

\begin{abstract}
As a common side effect of a variety of chemotherapy drugs, CINV severely limits the clinical use of chemotherapy drugs. Reducing the CINV levels may be useful to improve patient life quality and enable effective use of chemotherapy drugs. In this study, we investigated the protective effects of He-Wei granules (HWKL) against CINV using a cisplatin-induced $\left(5 \mathrm{mg} \mathrm{kg}^{-1}\right)$ pica model. HWKL consists of seven herbs: Pinellia ternata (Thunb.) Breit, Zingiber officinale Rosc, Panax ginseng C. A. Mey, Scutellaria baicalensis Georgi, Coptis chinensis Franch, Glycyrrhiza uralensis Fisch, and Ziziphus jujuba Mill. The CINV levels were measured based on the consumption of kaolin. Results showed that HWKL was able to reduce cisplatin-induced increase in kaolin consumption. Cisplatin-mediated increase in tryptophan hydroxylase1 (TPH1), TPH2, 5-hydroxytryptamine 3 receptors (5-HT 3 AR), neurokinin-1 receptors (NK-1R), $5-\mathrm{HT}_{3} \mathrm{AR}$ mRNA, preprotachykinin A (PPTA) mRNA, and NK-1R mRNA was markedly suppressed by HWKL. In addition, cisplatin-induced inhibition of monoamine oxidase (MAOA) and serotonin reuptake transporter (SERT) mRNA was significantly increased after HWKL treatment. HWKL dramatically enhanced appetite by regulating obestatin (OB) and ghrelin (GH); this contributed to further inhibit CINV. The results obtained for G protein-coupled receptor (GPR39) mRNA also confirmed the abovementioned conclusion. HWKL also contributed towards an increase in epidermal growth factor receptor (EGFR) and phosphorylation of extracellular regulated protein kinases ( $p$-ERK1/2) to help repair the damaged mucosa; this indicated that HWKL improved the gastrointestinal damage. Overall, our findings suggest that HWKL inhibit CINV by several mechanisms acting simultaneously and provide basic evidence to confirm that HWKL may be useful in clinical situations as a protective agent to prevent CINV. Moreover, 5-hydroxytryptamine (5-HT) and substance P (SP) result in inhibition of CINV.
\end{abstract}

\section{Introduction}

As a major global public health problem, cancer produces many severe effects and chemotherapy is still the major therapeutic option. ${ }^{1}$ However, due to severe toxic effects and complications, including CINV, nephrotoxicity, myelosuppression, cardiac damage, and hair loss, associated with chemotherapeutic drugs, patients suffer a lot and therapeutic effects of these drugs are reduced. ${ }^{2}$ Among the abovementioned adverse effects, CINV is considered to be the most unbearable because it leads to the development of complications such as fluid-electrolyte

${ }^{a}$ Development and Utilization Key Laboratory of Northeast Plant Materials, School of Traditional Chinese Materia Medica 48 Wenhua Road 103, Shenhe District, Shenyang 110016, China.E-mail:yinjun2002@ yahoo.com; Fax: +86-24-2398-6460; Tel: +86-24-2398-6491

${ }^{b}$ School of Chinese Materia Medica, Guangzhou University of Chinese Medicine, China $\dagger$ Electronic supplementary information (ESI) available. See DOI: 10.1039/c7ra06312a imbalance, weight loss, dehydration, anorexia, weakness, bone fracture, esophageal tear, prerenal azotemia, wound dehiscence, and decline in behavioral and mental ability. ${ }^{1,3}$ The side effects induced by CINV may affect patients' physical and mental status and their ability to look after themselves and function properly; this may lead to withdrawal of the anticancer treatment, which invariably affects treatment outcome and patient survival., ${ }^{\mathbf{1} 3}$ In addition, it increases patient anxiety and dissatisfaction with the hospital experience and may contribute to future anticipatory nausea. ${ }^{1,3}$ Although there are some treatments to combat CINV, their outcomes are unsatisfactory. ${ }^{4}$ Antiemetics commonly used for the prevention of CINV are expensive and can cause other side effects including diarrhea, headache, light-headedness, asthenia, fatigue, neutropenia, and hypotension. ${ }^{3}$ Therefore, there is an urgent need for more effective therapies or combination therapies to treat CINV in the treatment of cancer.

HWKL (patent number: ZL2011102359 15.7) was derived from the formulation of Ban-Xia Xie-Xin Tang or Ban-Xia Xie- 
Xin decoction (BXXXT), which was first described in Shang-HanLun about 1700 years ago for the treatment of many different gastrointestinal symptoms and treating patients with a weak spleen and stomach, internal stasis of heat and cold glomus, and fullness below the heart without pain, vomiting, and borborygmus..$^{5-10}$ Like BXXXT, HWKL consists of seven herbs: Pinellia ternata (Thunb.) Breit. (Pinelliae Rhizoma Praeparatum, Araceae, PR), Zingiber officinale Rosc. (Zingiberis Rhizoma Recens, Zingiberaceae, ZB), Panax ginseng C. A. Mey. (Ginseng Radix Et Rhizoma, Araliaceae, GR), Scutellaria baicalensis Georgi. (Scutellariae Radix, Labiatae, SR), Coptis chinensis Franch. (Coptidis Rhizoma, Ranunculaceae, CR), Glycyrrhiza uralensis Fisch. (Glycyrrhizae Radix Et Rhizoma Praeparata Cum Melle, Leguminosae, GHR), and Ziziphus jujuba Mill. (Jujubae Fructus, Rhamnaceae, JF). According to our previous study, ${ }^{11}$ HWKL could reduce high-dose cisplatin-induced nephrotoxicity and myelosuppression. A total of 37 major components, including ginsenosides, alkaloids, flavonoids, gingerols, and polysaccharides, were identified using UHPLC-Q-TOF-MS/MS, and 11 of these were confirmed and quantified using reference compounds. ${ }^{11}$

Furthermore, it is generally known that nausea usually leads to emesis and emesis occurs in $>90 \%$ of patients taking highly emetogenic chemotherapy drugs (such as cisplatin and carmustine) and 30-90\% of those taking moderately emetogenic chemotherapy drugs (for instance, irinotecan and oxaliplatin) without appropriate prophylaxis. ${ }^{\mathbf{1 , 4}}$ To study CINV in laboratory, several animal models, involving piglets, ferrets, and dogs, have been established. Due to their expense and difficult operating procedures, these animal models have not been widely used. Rats, one of the most common laboratory animals, have generally been considered unsuitable for studying CINV because they do not have a vomiting reflex. ${ }^{\mathbf{1 2}}$ However, although rats lack a motor reflex of emesis, they display atypical feeding behavior, termed pica response.$^{\mathbf{1 3 - 1 7}}$ Pica behavior is an aversive/ illness response that results in consumption of non-nutrient substances, such as kaolin, after an emetic stimulus. ${ }^{18,19}$ It has been confirmed that pica behavior can be used as a model of a nausea-like response or gastrointestinal malaise because it is induced by nausea stimuli and the amount of kaolin consumption is related to the severity of nausea in humans. ${ }^{13}$ In addition, it has been shown by the Japanese group Yamamoto et $a .^{13-16}$ that the magnitude of pica response in rats is directly related to the emetogenic potential in humans. The highly emetogenic anti-cancer drug (such as cisplatin)-induced kaolin ingestion behavior pica in rats is analogous to emesis. As an antineoplastic agent with high risk of emetogenic level increase, cisplatin was used to simulate CINV in our study, ${ }^{\mathbf{1 1 4}}$ and cisplatin-induced pica behavior was regarded as an acceptable model to measure the antiemetic effect of drugs according to kaolin consumption and other indices.,13,16,18,20

As a classical formula that has been used for more than 1000 years, BXXXT is considered to be effective in controlling the emesis levels. In our previous study, HWKL has been found to reduce kaolin intake of rats in a pica model. However, there have been no reports on the pharmacological effects and proper mechanism of action of HWKL on CINV; thus, our present study was aimed at the investigation of the protective effects and possible mechanisms of action of HWKL on CINV.

\section{Materials and methods}

\subsection{Reagents and chemicals}

Cisplatin for injection and ondansetron hydrochloride tablets from Qilu Pharmaceutical Co., Ltd (Shandong, China) were prepared in sterile saline to proper concentration. Domperidone tablets obtained from Xian Janssen Pharmaceutical Ltd. (Shanxi, China) were well distributed in pure water. Kaolin was purchased from Sinopharm Chemical Reagent Co., Ltd. The 5-HT enzyme-linked immunosorbent assay (ELISA) kit, SP, OB, GH, 5-hydroxyindole acetic acid (5-HIAA), TPH, MAOA, EGFR, and p-ERK1/2 ELISA kits were purchased from Shanghai Yuan-Mu bio-medical company (Shanghai, China). Malonaldehyde (MDA), superoxide dismutase (SOD), and glutathione peroxidase (GSH-PX) kit were obtained from Nanjing Jiancheng Bioengineering Institute (Nanjing, China). All other reagents were of highest purity and commercially available from Shenyang Lab Science and Trade Co., Ltd (Shenyang, China).

\subsection{Composition and preparation of samples}

All crude herbal drugs were purchased from Shenyang Guo-Da Pharmacy (Shenyang, Liaoning) and confirmed as genuine medicinal materials by Professor Jun Yin of the Pharmacognostical Department. All herbaria were stored in the School of Traditional Medicine. BXXXT and HWKL were manufactured according to ref. 15. Briefly, six crude herbal drugs (PR 12 g, ZR $9 \mathrm{~g}$, GR $9 \mathrm{~g}$, SR $9 \mathrm{~g}$, GHR $9 \mathrm{~g}$, and JF $6 \mathrm{~g}$ ) were used, and all, except $\mathrm{SR}$, were immersed in purified water for $2 \mathrm{~h}$ and decocted with 14 -fold amount of purified water for $2 \mathrm{~h}, 2 \times$. Coptidis Rhizoma $(\mathrm{CR}, 3 \mathrm{~g}$ ) was immersed in purified water for $2 \mathrm{~h}$ and decocted with 12 -fold purified water for $2 \mathrm{~h}, 3 \times$. After filtration, two decoctions were mixed and evaporated, spray dried with insoluble dextran into a granulated powder, and packaged and stored in foil containers at $4{ }^{\circ} \mathrm{C}(\mathrm{BXXXT})$; the crude herbal drugs (PR, Zingiberis Rhizoma Recens (ZB), GR, SR, GHR, and JF, ratio $6: 4: 3: 3: 3: 5)$ were immersed in purified water for $2 \mathrm{~h}$, except SR, and decocted with 14-fold amount of purified water for $2 \mathrm{~h}, 2 \times$. CR $(3 \mathrm{~g})$ was immersed in purified water for $2 \mathrm{~h}$ and decocted with 12 -fold purified water for $2 \mathrm{~h}, 3 \times$. After filtration, the two decoctions were mixed and evaporated, spray dried with insoluble dextran into a granulated powder, and packaged and stored in foil containers at $4{ }^{\circ} \mathrm{C}$ (HWKL). The BXXXT and HWKL granules were dissolved in pure water to a proper concentration before use. Kaolin diet preparation: kaolin pellets were prepared according to a previously reported method. ${ }^{16}$ Briefly, extra pure pharmacological-grade kaolin was mixed with $1 \%$ gum arabic in double-distilled water to form a thick paste. The kaolin paste was rolled and cut into small pieces resembling regular rat chow pellets through a $5 \mathrm{~mL}$ syringe. The pellets were completely dried at room temperature for nearly 4-5 days and stored in a sterile container at ambient temperature. 


\subsection{Animals and treatment}

Herein, 108 nine-week-old male Wistar rats weighing 250-280 g were purchased from Liaoning Changsheng biotechnology company (Benxi, China). The rats were kept in individual standard polypropylene transparent cages covered with steel grids under environmentally controlled conditions $\left(23 \pm 2{ }^{\circ} \mathrm{C}\right.$, relative humidity $50 \pm 10 \%, 12 \mathrm{~h}$ dark/light cycles, food and water ad libitum). ${ }^{21}$ For pica experiments, hydrated aluminum silicate mixed with acacia or gum arabic (Biotopped, Beijing, China) pellets was placed in adjacent separate compartments in a divided food hopper to ensure its regular accessibility throughout the experimental period. Sterile cage grills were used in each cage to prevent free access to non-nutritious substances such as bedding material (wood shavings) or stool. ${ }^{21}$ All experimental protocols were approved by the Animal Care and Use Committee of Shenyang Pharmaceutical University (SYPU-IACUC-C2016-0222-202). All experiments were performed in accordance with the published National Institutes of Health guidelines. Experiments commenced at least 7 days after the arrival of animals to the laboratory.

Rats were randomly divided into the following 9 groups $(n=$ 12): blank group, cisplatin group, cisplatin + ondansetron group, cisplatin + domperidone group, cisplatin + BXXXT group $\left(1.38 \mathrm{~g} \mathrm{~kg}^{-1}\right)$, cisplatin + HWKL low dose group $\left(1.18 \mathrm{~g} \mathrm{~kg}^{-1}\right)$, cisplatin + HWKL middle dose group $\left(2.36 \mathrm{~g} \mathrm{~kg}^{-1}\right.$, equal to the clinical dose of human beings), cisplatin + HWKL high dose group (4.725 $\mathrm{g} \mathrm{kg}^{-1}$ ), and HWKL group (2.36 $\mathrm{g} \mathrm{kg}^{-1}$ ). The clinical dose of BXXXT of human beings has been referenced in Shang-Han-Lun and other references. ${ }^{22-25}$ HWKL was used in the People's Liberation Army 463 Hospital for more than seven years. The clinical dose of HWKL for human beings was referenced to the dose used in the People's Liberation Army 463 Hospital. After 7 day acclimatization period, a measured quantity of kaolin pellets was filled in separate containers 3 days prior to the experiment to allow rats to psychologically adapt to its presence. After 3 days of habituation, rats were subjected to pica experiments. During the experimental observation period, rats in the blank and cisplatin groups were treated with pure water according to their bodyweight every day; the rats in the simple HWKL group were treated with HWKL $\left(2.36 \mathrm{~g} \mathrm{~kg}^{-1}\right.$, i.g. every day); rats in the cisplatin + ondansetron group were treated with ondansetron $\left(1.3 \mathrm{mg} \mathrm{kg}^{-1}\right.$, equal to the clinical dose of human beings, i.g. every day); rats in the cisplatin + domperidone group were treated with $3 \mathrm{mg} \mathrm{kg}{ }^{-1}$ domperidone (equal to clinical dose of human beings), i.g. every day; rats in the cisplatin + BXXXT group were treated with $1.38 \mathrm{~g} \mathrm{~kg}^{-1}$ BXXXT (equal to the clinical dose of human beings) i.g. every day; rats in cisplatin + HWKL low dose, cisplatin + HWKL middle dose, and cisplatin + HWKL high dose groups were treated with $1.18,2.36$, and $4.725 \mathrm{~g} \mathrm{~kg}^{-1}$ HWKL, respectively (equal to 0.5 -fold, 1-fold, and 2-fold clinical doses of human beings, respectively). The samples involved in the experiment were prepared in different concentrations to ensure that the same volume is administered. Cisplatin ( $5 \mathrm{mg} \mathrm{kg}^{-1}$, i.p.) was administered $1.5 \mathrm{~h}$ after each treatment with the antiemetic agent or its vehicle, except in the blank and HWKL groups.
Following the administration of cisplatin, kaolin consumption, bodyweight, and food intake were continuously observed every $24 \mathrm{~h}$ for $168 \mathrm{~h}$. Herein, 6 rats of each group were sacrificed after $72 \mathrm{~h}$ to obtain blood, serum, medulla oblongata, and ileum to measure the 5-HT relative targets such as 5-HT, 5-HIAA, TPH1, TPH2, MAOA, SERT mRNA, and 5- $\mathrm{HT}_{3} \mathrm{~A}$ mRNA. Other 6 rats of each group were sacrificed after $168 \mathrm{~h}$.

Kaolin consumption was used as the measurable parameter of vomiting and nausea according to the previous research. ${ }^{14-16,21,26-31}$ Food intake was used as the measurable parameter of anorexia.

\subsection{ELISA, MDA, GSH-PX, and SOD assays}

Serum: blood was drawn from the retro-orbital sinus $24 \mathrm{~h}, 72 \mathrm{~h}$, and $144 \mathrm{~h}$ after cisplatin injection and then centrifuged at $3500 \mathrm{rpm}$ for $15 \mathrm{~min}$ at $4{ }^{\circ} \mathrm{C}$ to obtain the serum.

Tissue homogenate: tissue sample was immersed in 9-fold volume of $\mathrm{PBS} / 0.9 \%$ saline and ground into a powder in ice by the Scientz-IID ultrasonic cell disruption system (Ningbo Scientz Biotechnology Co., Ltd, Ningbo, China). The extracts were centrifuged at $3500 \mathrm{rpm}$ for $15 \mathrm{~min}$ at $4{ }^{\circ} \mathrm{C}$ to remove insoluble materials.

The serum and supernatant fractions were analyzed using ELISA kits, MDA, GSH-PX, and SOD kits according to the manufacturer's instructions. After the experiment, the absorbance of each sample was measured at $450 \mathrm{~nm}$ (ELISA), $532 \mathrm{~nm}$ (MDA), $412 \mathrm{~nm}$ (GSH-PX), and $550 \mathrm{~nm}$ (SOD) using a microplate reader (Thermo Scientific L-117, USA).

Standard curves were prepared using diluted standard solutions to allow calculation of the relative target in the samples. All standards and samples were run in duplicate.

\subsection{Hematology and histopathology}

On the last experimental day (168 h), ileum and antrum were dissected, rinsed in ice-cold normal saline, and fixed in 10\% formalin for at least $24 \mathrm{~h}$. After fixation, all tissues were processed, paraplast-fixed blocks were sectioned (5 $\mu \mathrm{m}$ thickness), and slides were stained with haematoxylin and eosin. Representative images were obtained using a light microscope (Nikon, Japan).

\subsection{Quantitative real time PCR}

Total RNA was isolated from medulla oblongata, ileum, and antrum and detected by quantitative real-time PCR. Rat GAPDH was used as an endogenous control for sample normalization. Results are shown as fold increases relative to the expression of rat GAPDH. The PCR primer pairs were as follows: rat GAPDH (GenBank: NM-017008), 5'-GCAAGTTCAACGGCACAGTCA-3' (forward) and 5'-TGGTGGTGAAGA CGC CAGTAG-3' (reverse); rat SERT (GenBank: M79450), 5'-TAGATGCCGCCGCTC AGAT-3' (forward) and 5'-GTAGCCAAGCACCGTGAAGA-3' (reverse); rat 5- $\mathrm{HT}_{3} \mathrm{~A}$ receptor (GenBank: DQ226540), 5'-AGCTGGTGCA TAAGCAGGATT- $3^{\prime}$ (forward) and $5^{\prime}$-TCAGTCTTGTTGGCTTGGAAGG-3' (reverse); rat PPTA (GenBank: M34 184), 5' CAAGCCTCAGCAGTTCTTTGG-3' (forward) and 5'-GCGGACAC AGATGGAGATGAA-3' (reverse); rat NK-1 receptor (GenBank: 
NM-012667), $5^{\prime}$-G CAGCCCTTCTTATCCCTTGA-3' (forward) and $5^{\prime}$-GTCGCTCTACATTC CTCCACTT-3' (reverse); and rat GPR39, 5'-AGTGAGGAGAGCCGGACAG-3' (forward) and 5'-CAGTCATGT TTGGGTTTTGC- $3^{\prime}$ (reverse). The contents were measured by fold to control. The PCR primer sequence of rat GPR39 was provided by Beijing Protein Innovation Co., Ltd (Beijing, China).

\subsection{Immunoblotting assay}

The relative organ samples were lysed in pre-cold RIPA lysis buffer and centrifuged at $14000 \mathrm{rpm}$ for $15 \mathrm{~min}$ at $4{ }^{\circ} \mathrm{C}$. The total protein contained in the supernatants was separated by SDS-PAGE and transferred onto NC membranes. After blocking, the membranes were incubated overnight with relative primary antibodies. Next, the membranes were incubated with secondary antibodies and detected using an enhanced chemiluminescence detection kit (Nanjing Kengen Biotech Co., Ltd, Nanjing, Jiangsu, China). The intensity of each band was determined using the Gel-Pro32 software (Media Cybernetics, Bethesda, MD, USA).

$5-\mathrm{HT}_{3} \mathrm{~A}$ receptor antibody was purchased from abcam (Cambridge, MA, USA). NK-1 receptor antibody was purchased from ImmunoWay Biotechnology Company (Newark, DE, USA).

\subsection{Statistical analysis}

All values are presented as mean \pm SEM. One-way ANOVA analysis was used to compare the values obtained at the same time points, whereas two-way ANOVA analysis was used to compare the values obtained at different time points. According to the homogeneity of variance, LSD or Dunnett's T3 was chosen as the test method. Differences were considered statistically significance at $p<0.05$.

\section{Results}

\subsection{HWKL improves CINV and anorexia}

After cisplatin administration, food consumption and body weight were found to sharply reduce, whereas kaolin consumption was markedly increased in the cisplatin group in $24 \mathrm{~h}$ (Fig. 1). After cisplatin injection, anorexia and body weight loss were observed because of the severely reduced food intake and body weight in the cisplatin group in comparison with those in the blank group. HWKL can significantly reverse the adverse effects produced by cisplatin administration. Food consumption and body weight increased sharply in the HWKL treatment groups in comparison with those in the cisplatin group (Fig. 1).

According to the kaolin consumption of the cisplatin group, cisplatin administration caused acute and delayed emesis (Fig. 1A and B). As shown in Fig. 1A and B, a small amount of kaolin was consumed by rats in $-72 \mathrm{~h}$ because of their curiosity, but no kaolin was eaten by rats during the subsequent period. After cisplatin administration at $0 \mathrm{~h}, \mathrm{HWKL}$ dose-dependently (except in the first $24 \mathrm{~h}$ ) reduced kaolin consumption induced by cisplatin during the $168 \mathrm{~h}$ observation period, with significant reduction in each of the seven days in comparison with that in the cisplatin group. Kaolin consumption of the ondansetron group was found to be lower than that in any of the other groups after cisplatin injection at $24 \mathrm{~h}$ and increased steadily from $24 \mathrm{~h}$ to $72 \mathrm{~h}$, whereas it decreased in the other groups. A peak kaolin consumption in the cisplatin and ondansetron groups occurred at $72 \mathrm{~h}$, indicating delayed emesis although ondansetron contributed only slightly to it. Furthermore, HWKL treatment continuously reduced the kaolin consumption. Compared with those in the blank group, there were no significant differences in the food and kaolin consumption and body weight in the abovementioned groups; this suggested that HWKL alone did not affect the physical conditions (Fig. 1). Compared with that in the blank group, there was no significant difference in kaolin consumption in the HWKL group; this suggested that HWKL alone did not induce pica in rats (Fig. 1A and B).

\subsection{HWKL reduces acute CINV by regulating $5-\mathrm{HT}$ and its related biomarkers}

As shown in (A), (B), and (C) of Fig. 2, the 5-HT content in serum, medulla oblongata, and ileum of the cisplatin group was significantly increased as compared to that of the blank group at $24 \mathrm{~h}$; this indicated that cisplatin induced acute emesis through upregulating the content of 5-HT. Although the 5-HT content in the cisplatin group at $72 \mathrm{~h}$ increased sharply in comparison with that in the blank group, it was markedly lower as compared to that in the cisplatin group at $24 \mathrm{~h}$ (Fig. 2A). The 5-HT content in serum was markedly reduced in all groups after cisplatin injection at $24 \mathrm{~h}$ as compared to that in the cisplatin group; this indicated that the herbs (TCM) and drugs used in our study could inhibit additional 5-HT. However, the 5-HT serum content could still be significantly reduced at $72 \mathrm{~h}$ in three groups that were given different doses of HWKL as compared to that in the cisplatin group. Compared with that in the cisplatin group, the 5HT content in medulla oblongata and ileum of the three groups given different doses of HWKL was significantly reduced. Ondansetron was found to positively down-regulate the 5-HT content in the serum, medulla oblongata, and ileum (Fig. 2D and G). However, ondansetron failed to significantly reduce the 5-HT content in serum at $72 \mathrm{~h}$. Cisplatin inhibited the metabolism of 5HT because the 5-HIAA content in the serum and ileum was markedly reduced (Fig. 2B and H). HWKL was found to increase the metabolism of 5-HT, resulting in a significant increase in the 5-HIAA content in the serum and ileum (Fig. 2B and H). The herbs (TCM) and drugs used in this study failed to significantly control the 5-HIAA content in the medulla oblongata (Fig. 2E). As a consequence of an increased amount of 5-HT and a reduced amount of 5-HIAA, the ratio of 5-HIAA/5-HT sharply reduced in the serum, medulla oblongata, and ileum of the cisplatin group in comparison with that of the blank group. The ratios of 5-HIAA/ 5-HT in the groups given three different doses of HWKL were found to be higher than those in the cisplatin group; this showed that HWKL could positively control the level of 5-HT, keeping it at a normal level (Fig. 2C, F, and I).

According to the parts (E), (F), (G), and (H) of Fig. 3, the content of SERT mRNA in the medulla oblongata and ileum was reduced by cisplatin, whereas the $5-\mathrm{HT}_{3} \mathrm{AR}$ mRNA content in the 

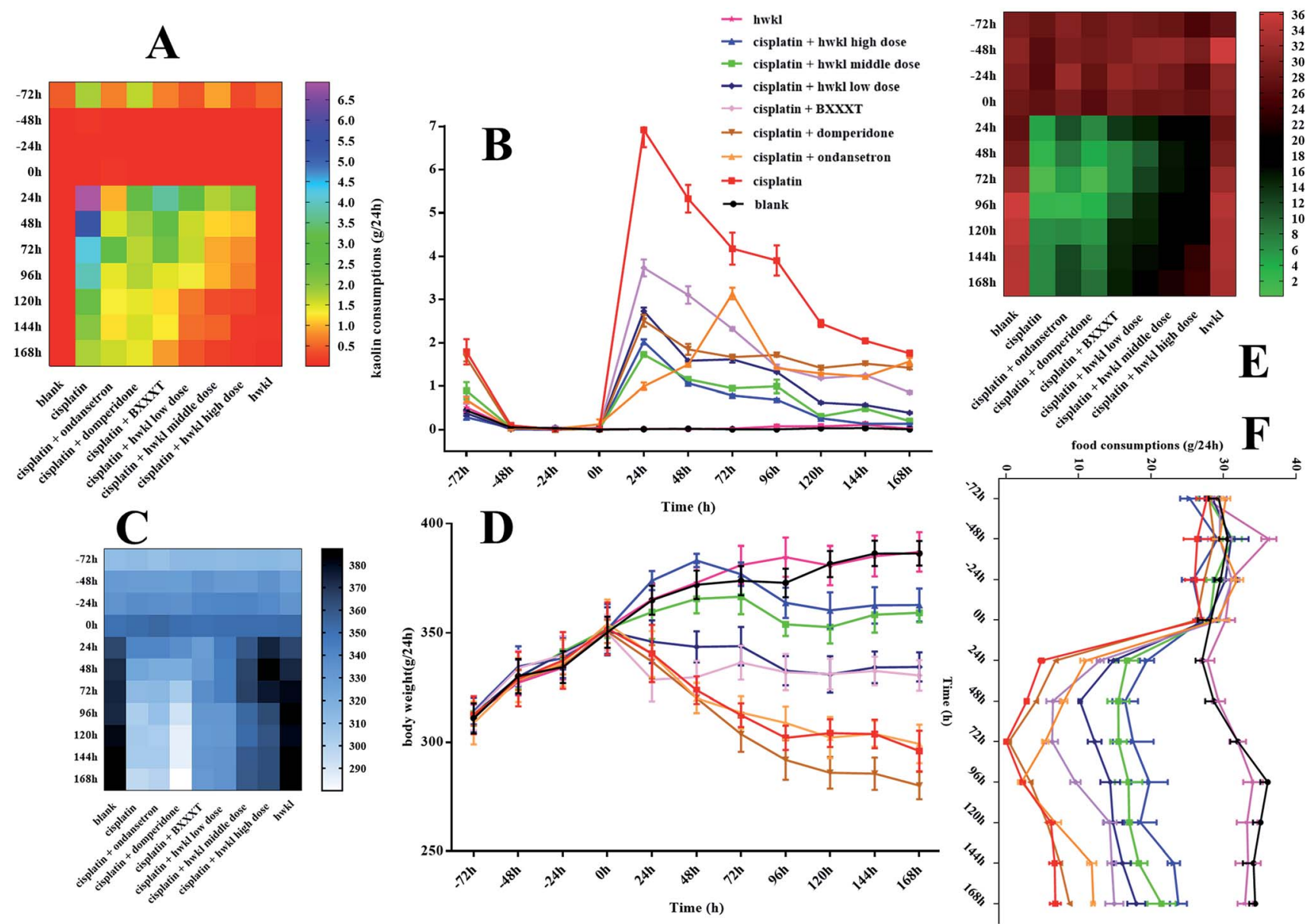

Fig. 1 Effect of HWKL on kaolin consumption, body weight, and food consumption at different time periods in cisplatin-treated rats ((A) heat map of kaolin consumption; (B) line graph of kaolin consumption; (C) heat map of body weight; (D) line graph of body weight; (E) heat map of food consumption; and (F) line graph of food consumption).

medulla oblongata and ileum increased following cisplatin administration in the cisplatin group. HWKL increased the content of SERT mRNA, whereas it reduced the $5-\mathrm{HT}_{3} \mathrm{AR}$ mRNA content. After cisplatin administration, the TPH- 1 content in the ileum and TPH-2 content in the medulla oblongata were found to sharply increase as compared to those in the blank group; this suggested that cisplatin could accelerate the synthesis of 5-HT by up-regulating the TPH content (Fig. 3C and D). HWKL can significantly reduce the content of TPH-1 and TPH-2 as compared to that in the cisplatin group. As a result of cisplatin administration, the MAO-A content in the ileum and medulla oblongata was reduced and inhibited in the cisplatin group as compared to that in the blank group (Fig. 3A and B), whereas HWKL increased the content of MAO-A to accelerate the metabolism of additional 5-HT caused by cisplatin administration. The relative content of $5-\mathrm{HT}_{3} \mathrm{AR}$ protein in the medulla oblongata and ileum of the cisplatin group increased significantly after cisplatin administration in comparison with that in the blank group, whereas HWKL treatment markedly reduced the content of $5-\mathrm{HT}_{3} \mathrm{AR}$ protein (Fig. 3J, K, and I). Ondansetron and domperidone positively regulated the content of MAO-A, but failed to regulate that of other biomarkers (Fig. 3).

\subsection{HWKL reduces delayed CINV by regulating SP and its related biomarkers}

As shown in (A), (B), and (C) of Fig. 4, the amount of SP in the serum, medulla oblongata, and ileum sharply increased after cisplatin administration as compared to that in the blank group. Furthermore, ondansetron and domperidone failed to significantly regulate the content of SP in the serum. Groups treated with HWKL after cisplatin administration were found to have less SP than the cisplatin group; this indicated that HWKL could reduce the level of emesis through down-regulating the SP content. The content of PPTA mRNA, which is the precursor of SP, increased sharply after cisplatin administration in the cisplatin group as compared to that in the blank group. HWKL treatment significantly reduced the PPTA mRNA content after cisplatin administration, whereas ondansetron, domperidone, and BXXXT failed to significantly reduce it (Fig. 4D and E). The same phenomenon was observed while regulating the NK-1R mRNA content and level of NK-1R protein expression (Fig. 4F-J). It is important to note that BXXXT and HWKL have a significant effect on down-regulating the NK-1R protein expression in the ileum (Fig. 4I and J). 

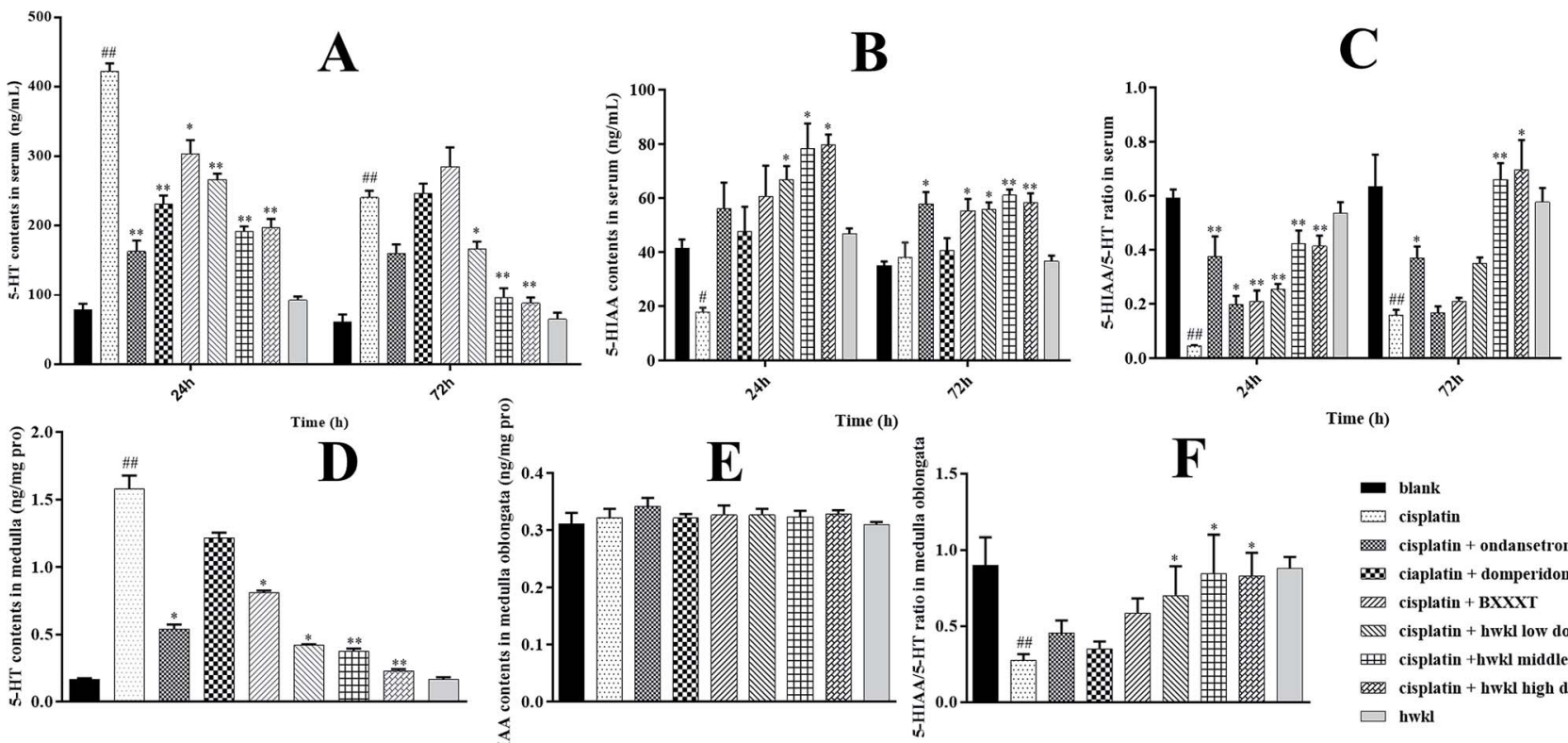

Time (h)
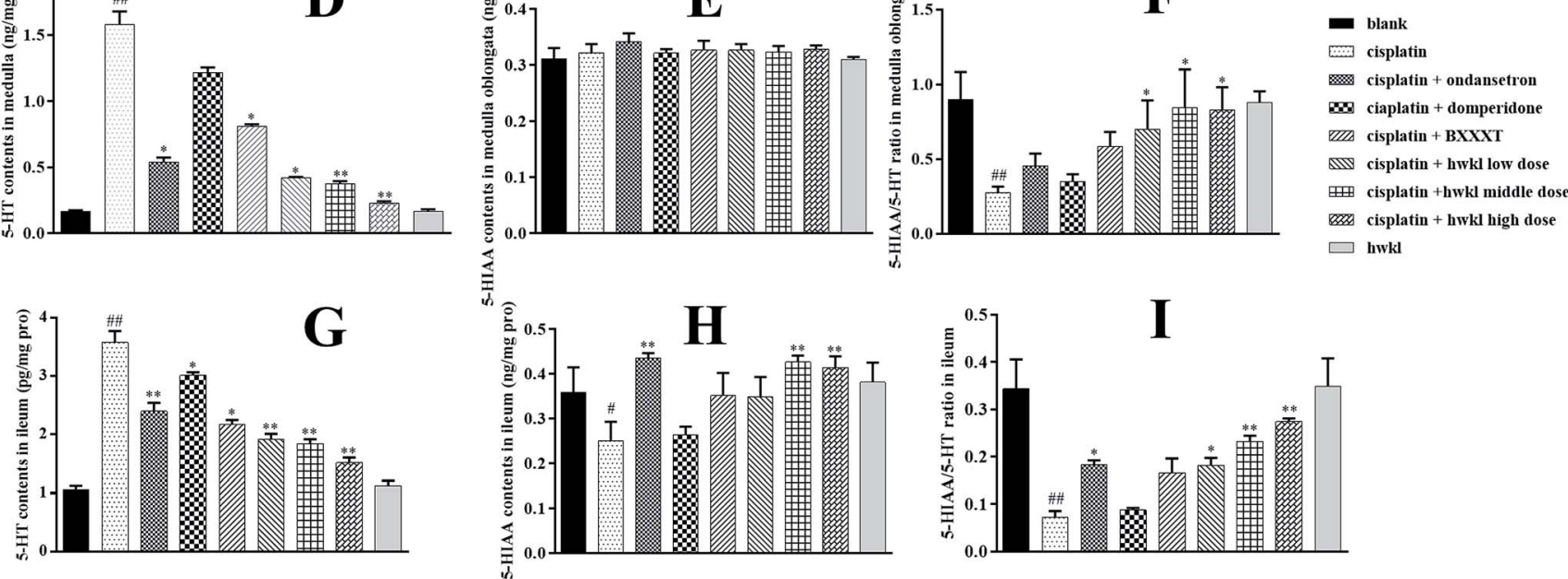

Fig. 2 Effect of HWKL on 5-HT content (A-C), 5-HIAA content (D-F), and 5-HIAA/5-HT ratio (G-I) in cisplatin-treated rat serum, medulla oblongata, and ileum.

The OB content in the serum and antrum of the cisplatin group increased sharply after cisplatin administration in comparison with that of the blank group (Fig. 5A and D). As demonstrated in (B), (C), (E), and (F) of Fig. 5, the GH content and the ratio of $\mathrm{GH} / \mathrm{OB}$ of the cisplatin group in the serum and antrum were significantly reduced after cisplatin injection in comparison with those of the blank group. Based on these results, cisplatin was considered to increase the $\mathrm{OB}$ content and reduce the amount of GH to inhibit the gastrointestinal function to aggravate CINV. Compared with that of the cisplatin group, the GH content in the serum was up-regulated in all groups except in the cisplatin + domperidone group at $72 \mathrm{~h}$. HWKL was able to simultaneously significantly increase the $\mathrm{GH}$ content and reduce the $\mathrm{OB}$ content, thus increasing the GH/OB ratio (Fig. 5). Furthermore, the GPR39 mRNA content in the antrum significantly increased in the cisplatin group after cisplatin administration as compared to that in the blank group; this indicated an increase in the OB receptors. HWKL efficiently reduced the marked increase in OB and GPR39 mRNA; this suggested that HWKL could combat the emesis induced by cisplatin administration by down-regulating the OB content and up-regulating the GH content.

\subsection{Effect of HWKL on gastrointestinal organ protection (stomach and small intestine)}

Histopathological examination of tissue sections obtained from the blank and HWKL groups did not show any tissue or cellular damage; this indicated that HWKL could not induce gastrointestinal injury. Moreover, the blank and HWKL groups exhibited normal histology and morphology (Fig. 6A and 7A). However, as shown in Fig. 6A, the cisplatin group exhibited severe injury to the gastric mucosa caused by cisplatin injection. Gastric mucosa necrosis and extra fibrous protein sclerosis were found in the cisplatin, ondansetron, domperidone, and BXXXT groups; moreover, edema and cell swelling and loss as well as degeneration due to vacuolization were observed in these groups. Congestion was seen in some areas as well as edema surrounding the capillaries. To objectively evaluate the protective effect of HWKL on the stomach, scores of histological slices of the stomach were obtained according to the pathological evaluations described by Yi et al. ${ }^{32}$ Details of the scores and their significance are shown in Fig. 6D. According to the results, it was confirmed that HWKL effectively protected the stomach from the damage caused by cisplatin administration.

In the blank and HWKL groups, the crypts in the glandular epithelium exhibited tight cylindrical structures and the lamina propria characterized by reticular connective tissue contained lymphatics and capillaries and was surrounded by individual lymphocytes, plasma cells, and eosinophils, as well as granulocytes that were not ungrouped (Fig. 7A). Moreover, high columnar absorptive cells stained with eosinophil were shown to have a regular arrangement. In addition, the villi were aligned in finger-like regular protrusions in the lumen, whereas the 

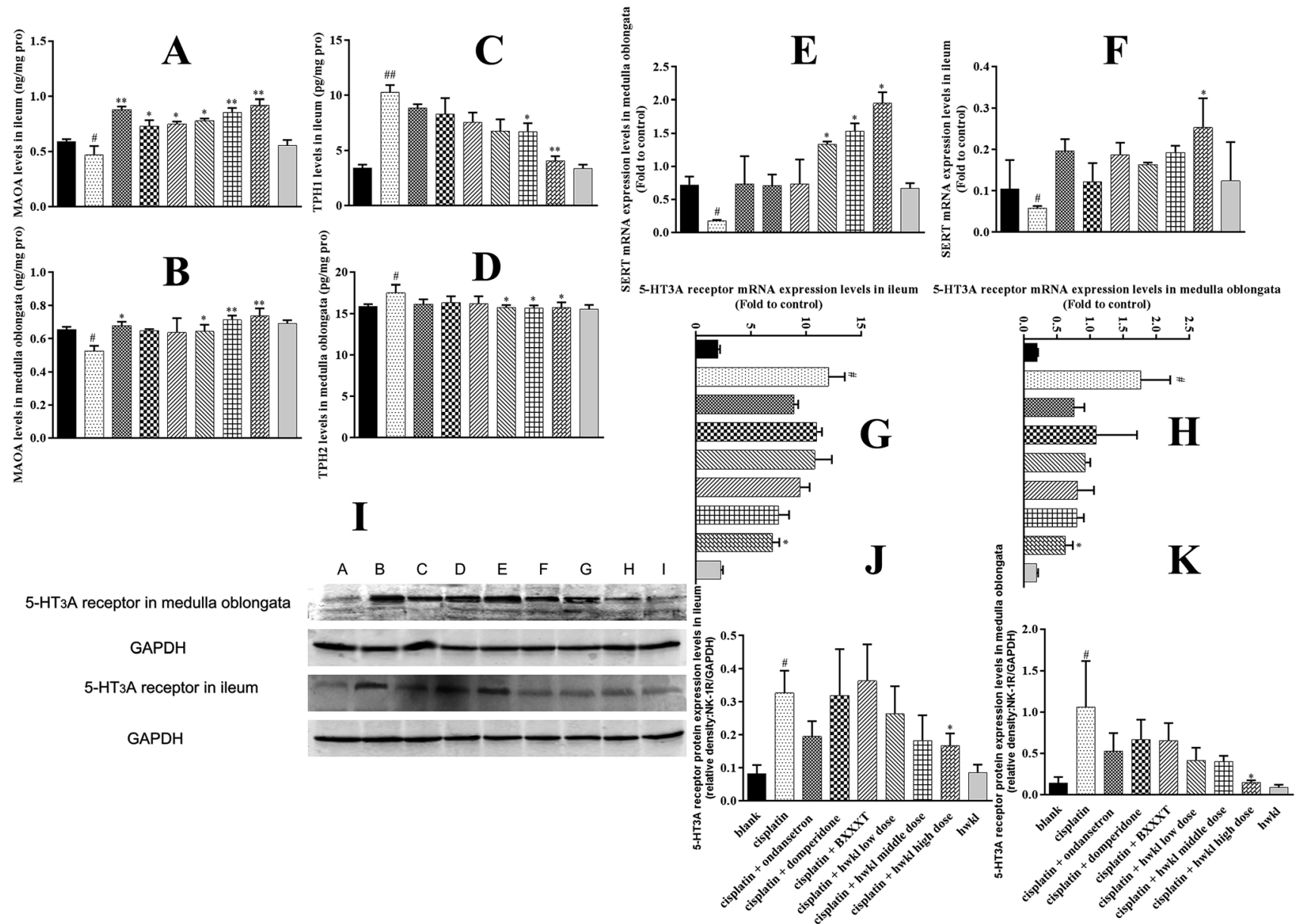

Fig. 3 Effect of HWKL on MAOA content ( $A$ and $B$ ) and TPH content ( $C$ and $D)$ in cisplatin-treated rat ileum and medulla oblongata. Effect of HWKL on SERT mRNA ( $F$ and $E$ ) and 5-HT3AR mRNA ( $G$ and $H$ ) content in cisplatin-treated rat ileum and medulla oblongata. Effect of HWKL on $5-\mathrm{HT}_{3} \mathrm{AR}$ protein content $(\mathrm{I}-\mathrm{K})$ in cisplatin-treated rat ileum and medulla oblongata.

goblet cells with oval nuclei were found with basophilic staining (Fig. 7A). However, abscised intestinal mucosa, inflammatory cell infiltration of the intestinal mucosa, and intestinal mucosa necrosis were clearly present in the cisplatin, ondansetron, domperidone, and BXXXT groups (Fig. 7A). In these groups, the crypts had lost their cylindrical and tightly arrayed structures, and their lumens were enlarged. Furthermore, the villi had lost their finger-like structure and were oval in shape; their length reduced due to the effect of edema, and denuded villi were observed. Dense cell swelling and degeneration due to vacuolization were observed in columnar cells at the apex. The goblet cells had lost their oval structures, and intense basophilic staining showed that they were secreting their mucosal content (Fig. 7A). However, HWKL effectively protected the ileum from the damage caused by cisplatin administration (Fig. 7A and B). Scores for the damaged ileum were given on the basis of capillary congestion, epithelial sloughing, edema and dilatation, crypt degeneration, denuded villi, and lymphocyte infiltration according to the pathological evaluations described by Aydin et $a l^{33}$ Details of the scores and their significance are given in Fig. 7B.

The content of EGFR and pERK1/2 in the cisplatin group increased significantly after cisplatin administration in comparison with those in the blank group; this suggested that extra EGFR and pERK1/2 were secreted to help recover the damaged tissue (Fig. $6 \mathrm{E}$ and F). Treatment with HWKL significantly contributed to the recovery of the damaged gastric mucosa via up-regulation of the EGFR and pERK1/2 content (Fig. 6E and F). BXXXT showed a tendency to increase the content of EGFR and pERK1/2, but there was no significant difference in comparison with that in the cisplatin group and ondansetron and domperidone showed very little effect (Fig. $6 \mathrm{E}$ and F).

As shown in (B) and (C) of Fig. 6 and (C) and (D) of Fig. 7, GSH-PX and SOD active levels of the antrum and ileum were found to be lowest in the cisplatin group as compared to those in the blank group. After HWKL administration, the GSH-PX and SOD active levels were found to be up-regulated; this indicated that HWKL could protect organs from the damage produced by cisplatin injection by reducing the oxidation level (Fig. 6 and 7). In addition, the content of MDA in the antrum and ileum decreased by HWKL treatment; this suggested that HWKL could reduce the oxidative level of the antrum and ileum after cisplatin injection (Fig. 6G and 7E). BXXXT had some effect on combatting the oxidative damage caused by cisplatin, 


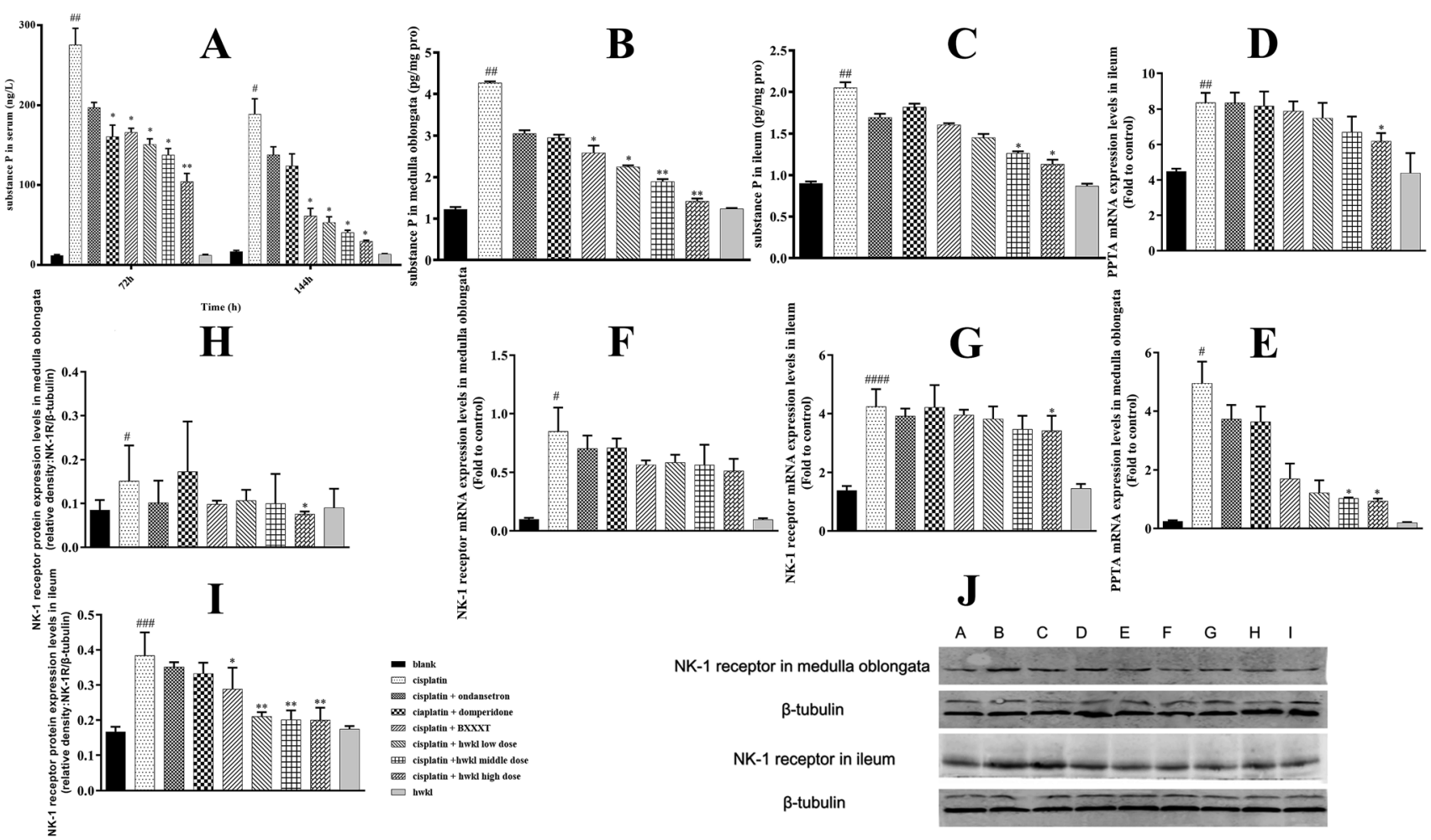

Fig. 4 Effect of HWKL on the substance P (SP) content in cisplatin-treated rat serum (A), medulla oblongata (B), and ileum (C). Effect of HWKL on PPTA mRNA ( $D$ and $E$ ) and NK-1R mRNA ( $F$ and $G$ ) contents in cisplatin-treated rat ileum and medulla oblongata. Effect of HWKL on NK-1R protein content (I, H, and $\mathrm{J})$ in cisplatin-treated rat ileum and medulla oblongata.

whereas ondansetron and domperidone had a very little effect on the same (Fig. 6 and 7).

\section{Discussion}

Nowadays, more and more research is being conducted on methods involving protection against chemotherapy-induced toxicity. ${ }^{20,21,34,35}$ Although many protective approaches are currently being evaluated, the protective effects of the agents used in these approaches are limited; this provided a rationale for developing combined strategies. ${ }^{35}$ Chinese herbal medicines are important for improving life quality through reducing chemotherapy-induced toxicity. In the present study, it is reported for the first time that systemic administration of HWKL, a product obtained using modern techniques and involving a modified ancient TCM formula, markedly reduces CINV. We successfully established a pica model to evaluate the protective effects of HWKL against CINV. HWKL attenuated the cisplatin-induced acute CINV by controlling the content of 5 -HT and related substances. HWKL controlled cisplatin-induced delayed CINV by regulating the level of SP and related substances. In addition, HWKL inhibited cisplatin-induced anorexia by regulating the levels of ghrelin and obestatin and reduced cisplatin-induced gastrointestinal oxidative stress. As a result, CINV was further inhibited because HWKL relieved cisplatin-induced gastrointestinal malaise. In addition, HWKL assisted the recovery of gastrointestinal damaged mucosa by regulating the levels of EGFR and pERK1/2; this further helped to inhibit CINV (Fig. 8).

Cisplatin has been shown to induce a pica response in rats, and a cisplatin model has been developed in which CINV occurring within the first 24 hours is defined as acute and that occurring more than 24 hours later is defined as delayed. ${ }^{18,19,36} \mathrm{It}$ has been reported that 3-6 $\mathrm{mg} \mathrm{kg}^{-1}$ cisplatin can induce significant pica behavior and gastrointestinal mucositis in rats. However, $6 \mathrm{mg} \mathrm{kg}^{-1}$ cisplatin cannot induce delayed vomiting because of severe anorexia. ${ }^{13}$ In our previous experiment, 3, 4, 5, and $6 \mathrm{mg} \mathrm{kg}^{-1}$ doses of cisplatin have been screened. According to the kaolin consumption, the general status of the animals, and the levels of biomarkers, a $5 \mathrm{mg} \mathrm{kg}^{-1}$ dose was considered to be more appropriate due to its stability and significance.

CINV is a major adverse effect of cytotoxic chemotherapy and is one of the reasons for patient non-compliance with cancer treatment. ${ }^{37,38}$ Nausea is defined as an unpleasant feeling in the upper gastrointestinal tract with an involuntary urge to vomit, and vomiting involves the involuntary and forceful expulsion of stomach content through the mouth. It has been reported that CINV is a complicated phenomenon with a sophisticated mechanism. The central nervous system and gastrointestinal tract have been reported to play key roles in CINV. Neurotransmitters such as 5-HT and SP are released after enterochromaffin cells are stimulated by cytotoxic chemotherapeutic agents, and these neurotransmitters have an effect on related receptors. Activated receptors act on the vagus nerve, and then 

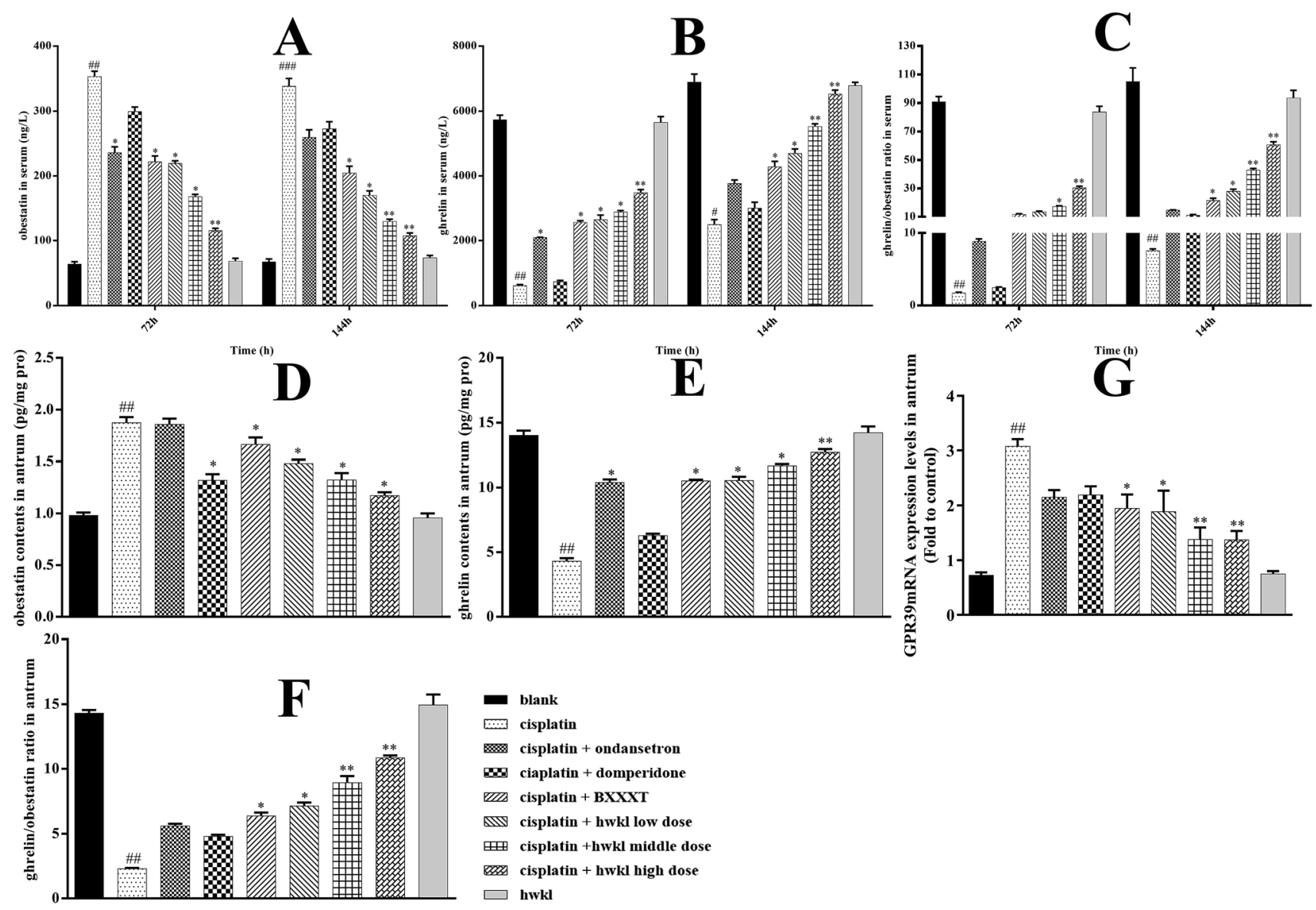

Fig. 5 Effect of HWKL on OB ( $A$ and $D)$ and GH (B and E) content, GH/OB ratio (C and F), and GPR39 mRNA (G) in cisplatin-treated rat serum and antrum. Serum used to test $\mathrm{GH}$ and $\mathrm{OB}$ was obtained at $72 \mathrm{~h}(n=12)$ and $144 \mathrm{~h}(n=6)$ after cisplatin administration.

the nerve impulse is transmitted to the vomiting center; as a consequence, vomiting is induced. In addition, nerve impulse can be transmitted to the chemoreceptor trigger zone (CTZ). 5-HT and SP, located in the CTZ, are released to the active related receptors, and then, the signals reach the vomiting center to induce emesis. Moreover, cytotoxic chemotherapeutic agents can directly affect the CTZ through blood circulation because CTZ is located in the fourth ventricle and there is no blood-brain barrier. ${ }^{13-16,37,38}$

In addition, gastrointestinal damage, occurring as a result of exposure to toxic chemotherapeutic agents, triggers the release of neurotransmitters which activate the chemoreceptors at the end of abdominal vagal afferents. Cisplatin-induced acute emesis has been confirmed to be related to the release of 5-HT, whereas SP release is considered to be the key factor in cisplatin-induced delayed emesis. In clinical situations, $5-\mathrm{HT}_{3}$ receptor antagonists, such as ondansetron and palonosetron, and NK1 receptor antagonists, such as aprepitant, are used alone or in combination with corticosteroids (for instance, dexamethasone) to treat CINV. Currently, antiemetic treatment involving $5-\mathrm{HT}_{3}$ and NK1 receptor antagonists is used to alleviate acute emesis and some delayed emesis, but delayed emesis remains an issue. ${ }^{37,38}$ However, the high cost and adverse effects of chemical antiemetic drugs cause extra pain to patients and impose a great burden. Therefore, it is important to explore novel strategies to inhibit CINV. ${ }^{4}$
CINV is a sophisticated reflection. Nowadays, it is considered that acute CINV is predominantly due to the release of $5-\mathrm{HT}$ within the gastrointestinal tract and nervous system, whereas SP plays an important role in delayed CINV. ${ }^{1,15,37-39}$ Unlike 5-HT and SP, OB and GH have been reported to be involved in CINV. ${ }^{\mathbf{4 0 - 4 4}}$ Tryptophan hydroxylase (TPH) catalyzes the ratelimiting step in the biosynthesis of 5-HT, and the expression level of TPH can be considered as a marker for 5-HT synthesis. Moreover, two kinds of TPH, TPH-1 and TPH-2, have been identified due to their distribution in the body. TPH-1 is responsible for 5-HT synthesis in non-neuronal cells, whereas TPH-2 is expressed in neurons of raphe nuclei and myenteric plexus. ${ }^{39,45}$ As the main metabolic enzyme of monoamines in rats, MAO can be divided into two hypo-types: MAO-A and MAO-B. 5-HT is mainly oxidized by MAO-A. ${ }^{37-39}$ As the metabolite of 5-HT, 5-HIAA is considered to be an important indicator in the metabolism of 5-HT, and nowadays, 5-HIAA/5-HT ratio is used more and more frequently to indicate the real level of the effect of a drug on 5-HT. SERT is the main carrier of 5-HT, whereas PPTA is the main source of SP. $5-\mathrm{HT}_{3} \mathrm{~A}$ is the most common form of 5-HT involved in chemotherapyinduced emesis, and NK-1R is the most compatible receptor of SP. In addition, it has been shown that SP helps increase the release of 5-HT from enterochromaffin cells. ${ }^{15}$ GPR39 is considered to be the receptor of $\mathrm{OB}$, and to measure the levels 

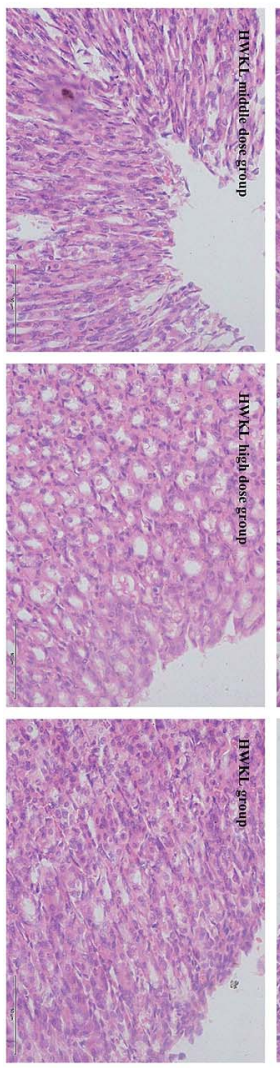

A
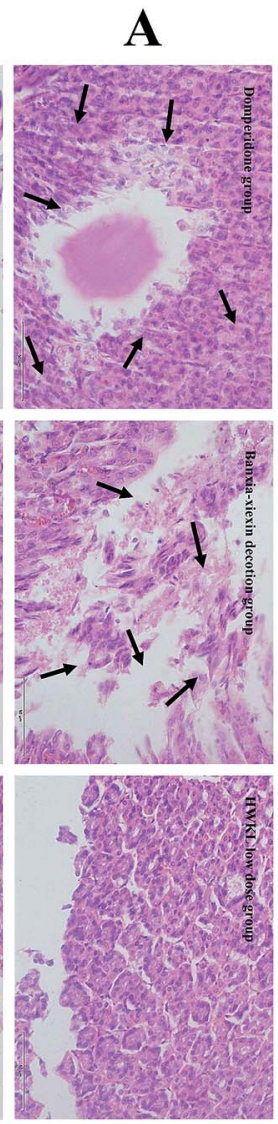
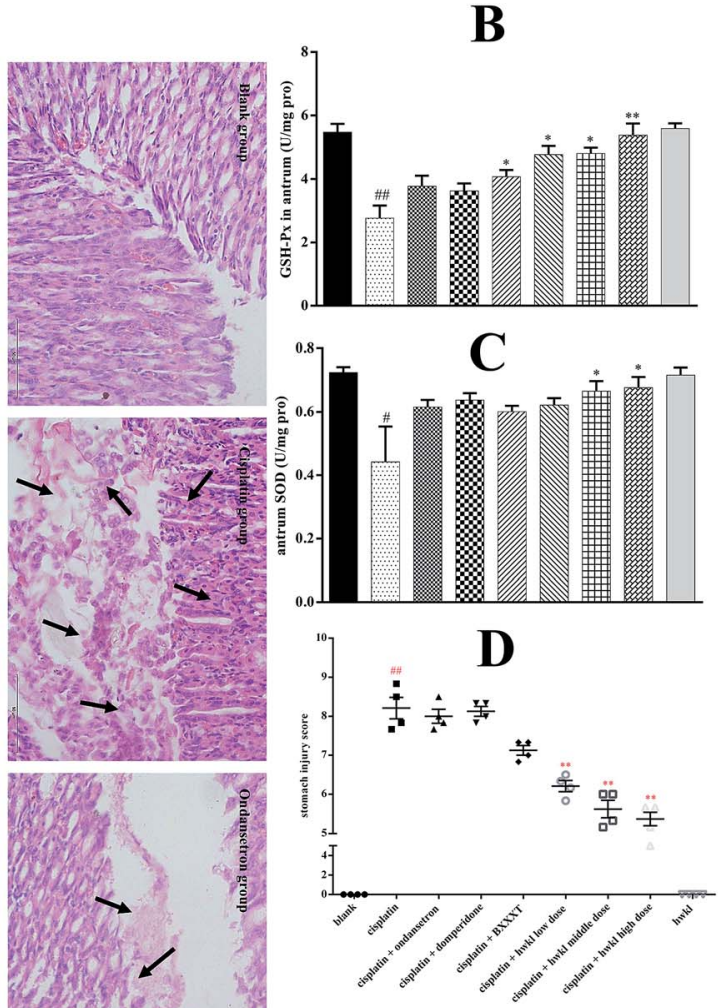

$\mathbf{E}$
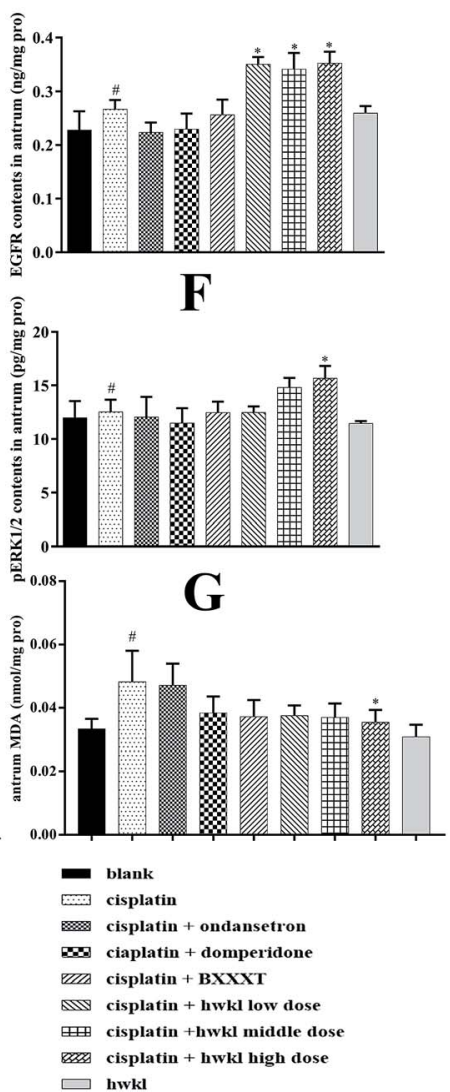

Fig. 6 Effect of HWKL on the protection of stomach (A) and GSH-PX (B), SOD (C), MDA (G), EGFR (E), and pERK1/2 (F) level in cisplatin-treated rats determined by ELISA, biochemical process, and histopathological diagnosis. Effect of HWKL on organ protection of stomach in cisplatin-treated rats by histopathological diagnosis based on inflammatory cells infiltration scoring (D).

of all these biomarkers, serum and key organs have been studied in this experiment. ${ }^{1,15,37-40,42-49}$

CINV is connected to gastrointestinal malaise, ${ }^{13}$ and cisplatin-induced gastrointestinal malaise including anorexia has been observed in our research. Therefore, reduction in cisplatin-induced gastrointestinal malaise may contribute to further inhibit chemotherapy-induced emesis. Regarding anorexia, GH and $\mathrm{OB}$, two different peptides that are encoded by the same preproghrelin gene, are considered to have an important role in its control. Stimulation of feeding and body weight gain are the main effects of GH. ${ }^{17,50,51}$ In addition, GH can improve the activity of motilin and gastrin to increase the rate of gastric motility by regulating the secretion of gastric acid and digestive enzymes. ${ }^{52}$ On the contrary, OB inhibits food intake and gastrointestinal motility. A previous study has shown that $\mathrm{OB}$ overexpression plays a significant role in the development of CINV.$^{41}$ GPR39 is considered to be the receptor of OB, and the level of GPR39 mRNA in the antrum is measured to examine the molecular relationship of $\mathrm{OB}$ at the gene level. According to the results obtained herein, we conclude that HWKL inhibits gastrointestinal damage by up-regulating GH and down-regulating $\mathrm{OB}$. As a consequence of the protective effect of HWKL on the gastrointestinal tract, CINV is further inhibited.
Cisplatin-induced oxidative stress is regarded as one of the main causes of gastrointestinal tract injury. Therefore, reduction of cisplatin-induced oxidative stress has an important effect on the inhibition of gastrointestinal injury; this can further contribute to the suppression of CINV. ${ }^{53}$

Recovery of damaged gastrointestinal mucosa helps to maintain the function of the gastrointestinal tract; thus, further advantages are associated with the inhibition of CINV. Epidermal growth factor (EGF) is considered to promote the repair and proliferation of impaired gastrointestinal mucosa following the action of cytotoxic drugs such as cisplatin. Selfdimerization and self-phosphorylation of EGFR will be activated by combination with related ligands, which can activate the EGFR/ERK signal transduction pathway. In addition, the Ras/Raf/MEK signal transduction pathway will be mediated and activated followed by activation of ERK phosphorylation. As a consequence, there is accelerated repair of the damaged mucosa.${ }^{54}$ Moreover, an increase in the phosphorylation status of ERK1/2 is regarded as a necessary factor for controlling the expression of PPTA and NK1 receptor mRNA.

In our experiment, we found a strange phenomenon that rats in the cisplatin + domperidone group were emaciated and weak and suffered mental fatigue. It is well known that domperidone can be used to treat gastric dysfunction and chemotherapy- 


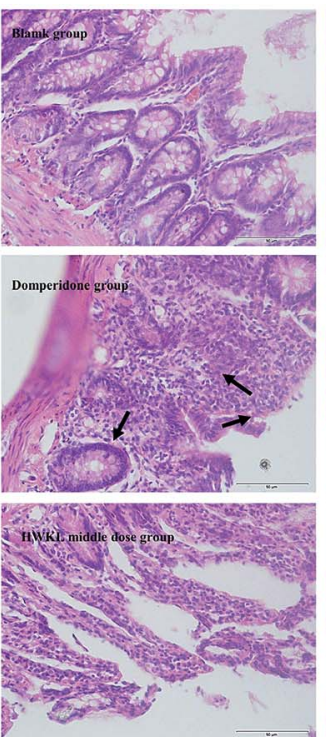

A
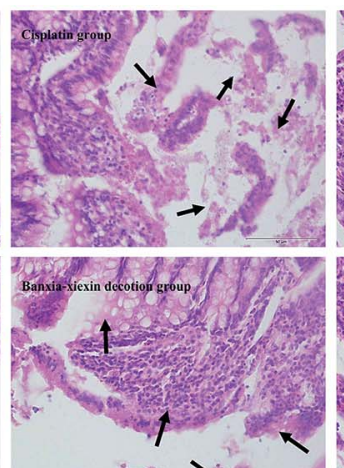

tanc
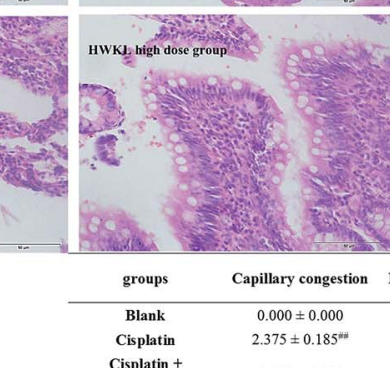

$B$

Cisplatin +

Cisplatin +

$\begin{array}{ll}\text { domperidone } & 2.125 \pm 0.172 \\ \text { isplatin + BXXXT } & 2.000 \pm 0.152\end{array}$

$2.000 \pm 0.152$ low dose

middle dose $\quad 1.542 \pm 0.142$ Cisplatin + HWKL

high dose

HWKL
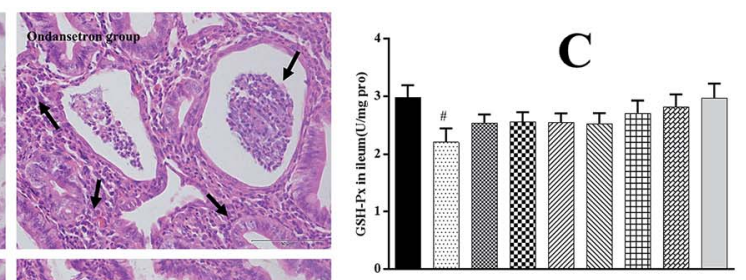

$\mathbf{E}$
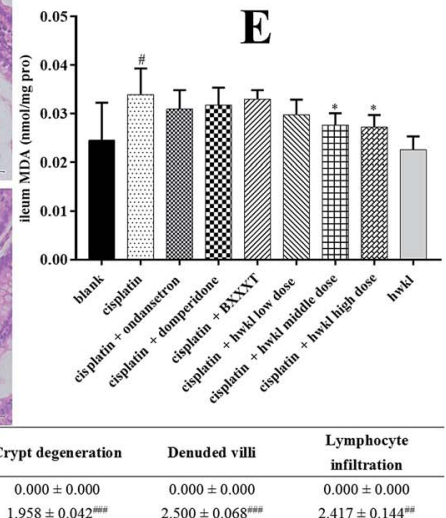

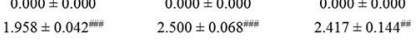

$1.875 \pm 0.080$

$1.917 \pm 0.250$

$2.292 \pm 0.142$

$2.208 \pm 0.158$

$2.500 \pm 0.204$

$2.542 \pm 0.142$

$2.167 \pm 0.068$

$1.833 \pm 0.118^{\circ}$

$2.583 \pm 0.160$

$2.042 \pm 0.080$

$1.792 \pm 0.125^{\circ}$

$1.625 \pm 0.042$

$1.500 \pm 0.226$

$1.625 \pm 0.185^{*}$

$1.583 \pm 0.173^{\circ}$

$.417 \pm 0.198^{\circ}$

$0.000 \pm 0.000$

$1.458 \pm 0.229^{*}$

$0.000 \pm 0.000$

$1.375 \pm 0.249$

$1.250 \pm 0.107^{*}$

$0.000 \pm 0.000$

$2.208 \pm 0.142$

$2.333 \pm 0.215$

$2.000 \pm 0.068$

$1.833 \pm 0.068^{*}$

$1.792 \pm 0.042$

$1.542 \pm 0.172^{*}$

$1.500 \pm 0.180$

$1.458 \pm 0.105^{*}$

$1.417 \pm 0.108^{*}$

$0.000 \pm 0.000$

Fig. 7 Effect of HWKL on the protection of small intestine (ileum) (A) and GSH-PX (C), SOD (D), and MDA (E) level in cisplatin-treated rats determined by biochemical process and histopathological diagnosis. Histopathological evaluation of cisplatin-induced small intestine injury (B).

induced emesis and nausea. However, domperidone was not effective in antagonising cisplatin-induced toxicity in this study. We believed that reduction in kaolin consumption in the cisplatin + domperidone group was not due to the efficiency of domperidone but because rats did not have enough energy to eat. However, this phenomenon requires further study.

Moreover, seven herbs are included in HWKL. Among them, $\mathrm{PR}$ and $\mathrm{ZB}$ are considered to be the main components on the basis of the traditional Chinese medicine theory. It has been

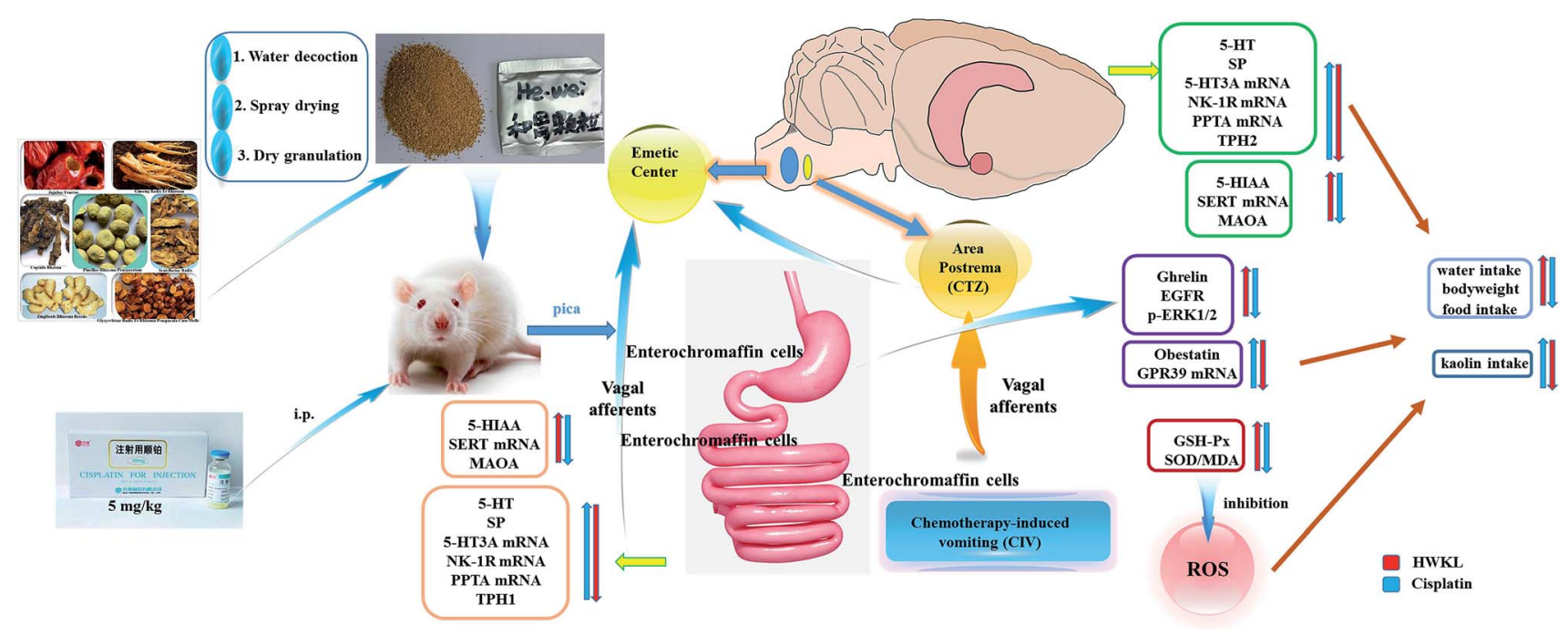

Fig. 8 Herbs involved in HWKL and proposed pathway of HWKL inhibiting chemotherapy-induced vomiting in rats. 
reported that a water decoction of $\mathrm{PR}$ and $\mathrm{ZB}$ can inhibit cisplatin-induced pica by down-regulating the levels of obestatin. ${ }^{6,20,41} 6$-Gingerol in $\mathrm{ZB}$ is found to be effective in controlling CINV. ${ }^{55-57}$ GR is a well-known TCM that is believed to be the king of herbs in the Orient, especially in China, Korea, and Japan. Ginsenosides and polysaccharides are considered to be active constituents with anticancer, antioxidant, and immunomodulation effects, which contribute to enhance immunomodulation effect against CINV. ${ }^{21,58}$ Chrysin has been reported to attenuate cisplatin-induced colon toxicity via amelioration of oxidative stress. ${ }^{59}$ Its glucosides are present in HWKL. ${ }^{11}$ Baicalin in SR and berberine in CR are often used in clinical situations to treat gastrointestinal inflammation and have been reported to treat cancer. ${ }^{2,58,60,61}$ Chemical compositions in HWKL can work synergistically in regulating the biomarkers related to CINV. As a result, CINV is inhibited.

\section{Conclusion}

Overall, multiple pathways are responsible for the inhibition of CINV according to our results. Simultaneous inhibition of various signaling pathways involved in CINV may be necessary for production of a therapeutic effect by a drug or TCM. Our results demonstrate that HWKL needs to be further studied to identify its exact mechanisms of action and active compounds responsible for the inhibition of CINV.

\section{Conflicts of interest}

The authors of this manuscript state that they have no conflicts of interest.

\section{References}

1 P. Hesketh, N. Engl. J. Med., 2008, 358, 2482-2494.

2 F. Qi, BioSci. Trends, 2010, 4, 297-307.

3 R. Haniadka, S. Popouri, P. L. Palatty, R. Arora and M. S. Baliga, Integr. Cancer Ther., 2012, 11, 18-28.

4 L. Schwartzberg, Expert Rev. Pharmacoeconomics Outcomes Res., 2014, 14, 825-834.

5 H. Lu, S. Ma, Y. Guo, J. Cai, X. Wang and W. Ye, Chin. Arch. Tradit. Chin. Med., 2009, 27, 1082-1084.

6 H. X. Yu and D. S. Wang, Chin. Arch. Tradit. Chin. Med., 2009, 27, 1537-1540.

7 R. Xue, Y. Cao, N. Han, X. Lin, Z. Liu and J. Yin, J. Ethnopharmacol., 2011, 137, 1156-1160.

8 L. Q. Zhao, S. S. Zhang, Z. F. Wang and A. Wiebrecht, Dtsch. Z. Akupunkt., 2013, 56, 30-31.

9 Y. Li, B. Zhang, Y. Lai and Z. Chen, Chin. J. Exp. Tradit. Med. Form., 2014, 20, 180-184.

10 G. Chen, Y. Yang, M. Liu, Z. Teng, J. Ye, Y. Xu, X. Cai, X. Cheng, J. Yang, C. Hu, M. Wang and P. Cao, J. Ethnopharmacol., 2015, 166, 149-156.

11 Z. Song, H. Chang, N. Han, Z. Liu, Y. Liu, H. Wang, J. Shao, Z. Wang, H. Gao and J. Yin, RSC Adv., 2017, 7, 19794-19807.
12 C. C. Horn, B. A. Kimball, H. Wang, J. Kaus, S. Dienel, A. Nagy, G. R. Gathright, B. J. Yates and P. L. R. Andrews, Biotechnol. Prog., 2013, 31, 1433-1441.

13 K. Yamamoto, S. Tatsutani and T. Ishida, Front. Pharmacol., 2016, 7, 534.

14 K. Yamamoto, M. Nakai, K. Nohara and A. Yamatodani, Eur. J. Pharmacol., 2007, 554, 34-39.

15 K. Yamamoto, K. Asano, A. Tasaka, Y. Ogura, S. Kim, Y. Ito and A. Yamatodani, Br. J. Pharmacol., 2014, 171, 2888-2899.

16 K. Yamamoto, K. Asano, N. Matsukawa, M. Imaizumi and A. Yamatodani, J. Pharmacol. Toxicol. Methods, 2011, 63, 30-34.

17 A. M. Holmes, J. A. Rudd, F. D. Tattersall, Q. Aziz and P. L. R. Andrews, Br. J. Pharmacol., 2009, 157, 865-880.

18 N. Takeda, S. Hasegawa, M. Morita and T. Matsunaga, Pharmacol. Biochem. Behav., 1993, 45, 817-821.

19 R. J. Mccaffrey, Physiol. Behav., 1985, 35, 151-156.

20 A. K. Pillai, K. K. Sharma, Y. K. Gupta and S. Bakhshi, Pediatr. Blood Canc., 2011, 56, 234-238.

21 R. Sathyanath, B. R. H. Rao, H.-G. Kim, J.-H. Cho and C.-G. Son, Pharmaceut. Biol., 2013, 51, 1052-1060.

22 Z. Song, H. Chang, N. Han, Z. Liu, Y. Liu, H. Wang, J. Shao, Z. Wang, H. Gao and J. Yin, RSC Adv., 2017, 7, 19794-19807.

23 G. Chen, Y. Yang, M. Liu, Z. Teng, J. Ye, Y. Xu, X. Cai, X. Cheng, J. Yang and C. Hu, J. Ethnopharmacol., 2015, 166, 149-156.

24 Y. Wang, R. Xu, J. Xiao, J. Zhang, X. Wang, R. An and Y. Ma, J. Pharmaceut. Biomed. Anal., 2014, 88, 525-535.

25 R. Xue, Y. Cao, N. Han, X. Lin, Z. Liu and J. Yin, J. Ethnopharmacol., 2011, 137, 1156.

26 H. H. Aung, L. Dey, S. Mehendale, J. T. Xie, J. A. Wu and C. S. Yuan, Canc. Chemother. Pharmacol., 2003, 52, 453-458.

27 H. H. Aung, S. R. Mehendale, J. T. Xie, J. Moss and C. S. Yuan, Life Sci., 2004, 74, 2685-2691.

28 P. A. Cabezos, G. Vera, M. I. Martin-Fontelles, R. FernandezPujol and R. Abalo, Neurogastroenterol. Motil., 2010, 22, 797-e225.

29 Y. L. Liu, N. Malik, G. J. Sanger, M. I. Friedman and P. L. R. Andrews, Physiol. Behav., 2005, 85, 271-277.

30 R. J. Mccaffrey, Physiol. Behav., 1985, 35, 151-156.

31 K. Yamamoto, M. P. Ngan, N. Takeda, A. Yamatodani and J. A. Rudd, Physiol. Behav., 2004, 83, 151-156.

32 S. X. Yi, Y. Du, Y. P. Lin, J. B. Hong, H. Peng and Y. Huang, China J. Tradit. Chin. Med. Pharm., 2010, 25, 1462-1467.

33 I. e. a. Aydin, Eur. Rev. Med. Pharmacol. Sci., 2014, 2076-2083. 34 L. Guo, S. P. Bai, L. Zhao and X. H. Wang, Med. Oncol., 2012, 29, 1656-1662.

35 Z. Y. Teng, X. L. Cheng, X. T. Cai, Y. Yang, X. Y. Sun, J. D. Xu, W. G. Lu, J. Chen, C. P. Hu, Q. Zhou, X. N. Wang, S. L. Li and P. Cao, Sci. Rep., 2015, 5, 15592.

36 J. Shi, Oncol. Lett., 2014, 8, 2017-2022.

37 C. Rojas, M. Raje, T. Tsukamoto and B. S. Slusher, Eur. J. Pharmacol., 2014, 722, 26-37.

38 C. Rojas and B. S. Slusher, Eur. J. Pharmacol., 2012, 684, 1-7. 39 N. van Lelyveld, J. Ter Linde, M. E. Schipper and M. Samsom, Neurogastroenterol. Motil., 2007, 19, 342-348. 
40 K. Ataka, A. Inui, A. Asakawa, I. Kato and M. Fujimiya, Am. J. Physiol. Gastrointest. Liver Physiol., 2008, 294, G1210-G1218.

41 Q. Qian, W. Chen, C. Guo, W. Wu, W. Qian and S. Li, J. Ethnopharmacol., 2011, 135, 186-193.

42 U. Gurriaran-Rodriguez, I. Santos-Zas, O. Al-Massadi, C. S. Mosteiro, D. Beiroa, R. Nogueiras, A. B. Crujeiras, L. M. Seoane, J. Senaris, T. Garcia-Caballero, R. Gallego, F. F. Casanueva, Y. Pazos and J. P. Camina, J. Biol. Chem., 2012, 287.

43 A. Stengel and Y. Tache, Journal of Neurogastroenterology and Motility, 2012, 18, 138-149.

44 X.-J. Su, R.-X. Dong, Y.-P. Li, S.-G. Yang and Z.-F. Li, Peptides, 2014, 52, 58-60.

45 N. Carkaci-Salli, U. Salli, K. L. Kuntz-Melcavage, M. M. Pennock, H. Ozgen, I. Tekin, W. M. Freeman and K. E. Vrana, Brain Res. Bull., 2011, 84, 376-380.

46 A. Asakawa, K. Ataka, K. Fujino, C.-Y. Chen, I. Kato, M. Fujimiya and A. Inui, J. Gastroenterol. Hepatol., 2011, 26, 73-74.

47 A. J. Agnew, E. Robinson, C. M. McVicar, A. P. Harvey, I. H. A. Ali, J. E. Lindsay, D. M. McDonald, B. D. Green and D. J. Grieve, Br. J. Pharmacol., 2012, 166, 327-338.

48 X. Chen, K. J. Margolis, M. D. Gershon, G. J. Schwartz and J. Y. Sze, PLoS One, 2012, 7, e32511.

49 L. S. Sen, B. Karakoyun, C. Yegen, M. Akkiprik, M. Yuksel, F. Ercan, A. Ozer and B. C. Yegen, Peptides, 2015, 71, 8-19.
50 B. Yu, Y. Hu, Y. Zhao, X. Yu, J. Xu, Z. Hua and Z. Zhao, Support. Care Canc., 2017, 25, 1651-1659.

51 S. O. Fetissov, N. Lucas and R. Legrand, Front. Endocrinol., 2017, 8, 10.

52 C. Y. e. a. Geng, Acta Vet. Zootech. Sin., 2013, 44, 829-836.

53 G. J. Dugbartey, L. J. Peppone and I. A. de Graaf, Toxicology, 2016, 371, 58-66.

54 H. Zhang, H. Guo, Y. C. Zhang, M. Liu, K. Ai, Y. M. Su, M. H. Li and T. L. Li, Acupunct. Res., 2014, 39, 351-357.

55 F. Fahimi, K. Khodadad, S. Amini, F. Naghibi, J. Salamzadeh and S. Baniasadi, Iran. J. Pharm. Res. (IJPR), 2011, 10, 379384.

56 W. M. Marx, L. Teleni, A. L. Mccarthy, L. Vitetta, M. K. Dan, D. Thomson and E. Isenring, Nutr. Rev., 2013, 71, 245-254.

57 A. N. Nasrabadi, Tehran Univ. Med. J., 2013, 71, 395-403.

58 F. Qi, L. Zhao, A. Zhou, B. Zhang, A. Li, Z. Wang and J. Han, BioSci. Trends, 2015, 9, 16-34.

59 R. Khan, A. Q. Khan, W. Qamar, A. Lateef, M. Tahir, M. U. Rehman, F. Ali and S. Sultana, Toxicol. Appl. Pharmacol., 2012, 258, 315-329.

60 L. Y. Du, D. W. Qian, E. X. Shang, P. Liu, S. Jiang, J. M. Guo, S. L. Su, J. A. Duan, J. Xu and M. Zhao, J. Ethnopharmacol., 2015, 169, 156-162.

61 W. Zhang, M. W. Saif, G. E. Dutschman, X. Li, W. Lam, S. Bussom, Z. Jiang, M. Ye, E. Chu and Y.-C. Cheng, J. Chromatogr. A, 2010, 1217, 5785-5793. 